\title{
Do we need an age specific measure of consensual poverty for older adults? Evidence from the poverty and social exclusion survey
}

\author{
Lucy K Smith, Ruth M Hancock
}

J Epidemiol Community Health 2004;58:616-617. doi: 10.1136/jech.2003.011544

$\mathrm{R}$ esearch on health inequalities among older adults is hampered by the lack of a suitable measure of poverty in this age group. ${ }^{12}$ Grundy and Holt ${ }^{1}$ have recommended the development of a measure for studying health inequalities in older people, which combines basic living conditions and social customs ${ }^{3}$ with household resources. The 1999 poverty and social exclusion omnibus survey (PSEOS) ${ }^{4}$ yields data that have been used to devise such a measure based on the consensual approach, that is "deprivation from goods, services and activities which the majority of the population defines as being the "necessities' of modern life". ${ }^{4}$ Some exploration of age variations in the perceptions of necessities has been carried out by Gordon et al focusing on differences among 16 to 24 year olds. Here we extend the use of PSEOS data to establish whether older and younger adults agree on consensual measures of poverty.

\section{PARTICIPANTS, METHODS, AND RESULTS}

The 1999 PSEOS asked a representative sample of British people aged 16 and over and living in private households ( 1855 respondents) to determine which items from a list of 54 "all adults should be able to afford and which they should not have to do without"1 classifying them as "necessary" or "not necessary". The PSEOS categorised items as necessities where more than $50 \%$ of respondents identified them in this way.

We explored respondents' views on the 54 goods, services, and activities (see appendix on the journal web site http:// www.jech.com/supplemental) by age (16-64 years; 65 or more years), calculating the percentage of respondents identifying each item as necessary. Ranks of items were plotted for older adults compared with younger adults. As the threshold of $50 \%$ for a necessity used in the original study was arbitrary, comparisons of the items included based on older or younger adults were made informally and the change in ranks observed but not formally tested.

For all respondents, using the PSEOS threshold of 50\%, 35 items were classified as necessities, with beds and bedding ranked highest. Nineteen items and activities were not classified as necessities. These were predominantly consumables; satellite television and access to the internet were ranked the lowest. The appendix lists these items in ranked order for all respondents.

Of the 54 items, older people were more likely to identify 34 of them as a necessity, and younger people were more likely to identify 12 of them as a necessity. The biggest percentage differences were seen among consumables (dressing gown, television, and two pairs of all weather shoes), which older people were more likely to classify as necessary. Figure 1 illustrates the differences in rankings of items between older and younger adults. There was less variability in lower ranked items, but slightly more among the higher ranks (necessities). However applying the PSEOS threshold of $50 \%$, would make little change to the items included if necessities were defined separately for older adults. The main difference between the age groups was the percentage that rated each item as necessary, rather than the order in which items were ranked. In only a minority of cases did this affect whether items were classified as a necessity. When compared with all respondents, two additional "necessities" were identified by older people: "new not second hand clothes" and a dressing gown. For those under 65 , one fewer necessity ("an outfit for social occasions") was identified.

Sensitivity analyses using different age groupings showed similar results. The largest difference was seen among the 16 to 24 year old age group where respondents were less likely to rank items as necessities as discussed by Gordon et al. ${ }^{5}$ If these young adults are excluded, and adults aged 25 to 64 years are compared with those aged 65 years and over, the small change in rankings we have shown in figure 1 diminish further.

\section{COMMENT}

To our knowledge this is the first study to explore age related differences in a consensual measure of poverty. We conclude that the consensual definition of poverty is similar for older and younger adults and therefore may be a useful measure

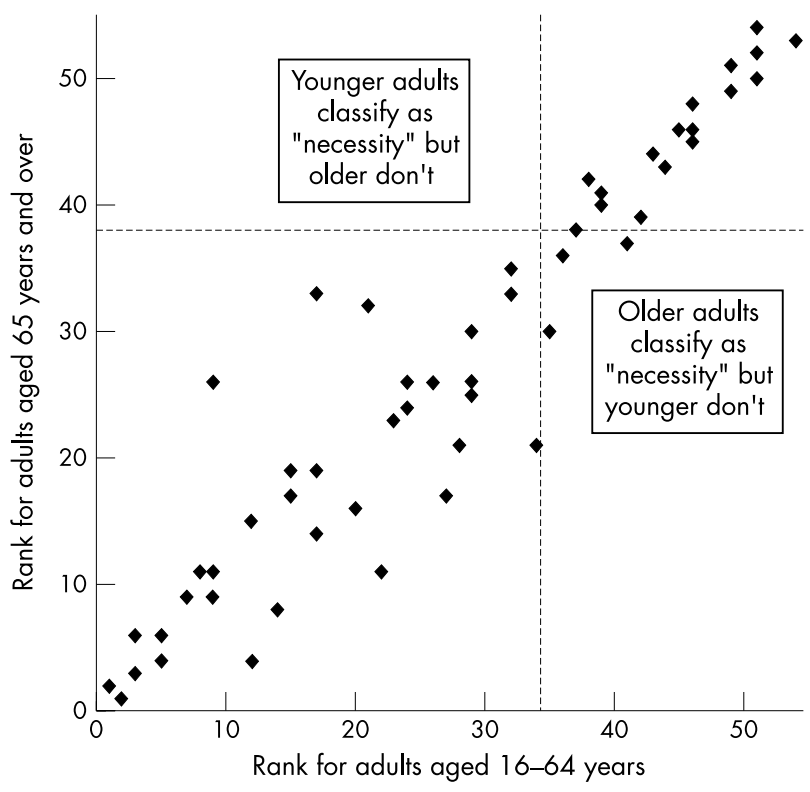

Figure 1 Rank of items for adults aged 16 to 64 years compared with rank of items for adults aged 65 years and over. Rank 1 is highest ranked item, rank 54 lowest ranked item. Dotted lines indicate $50 \%$ of respondents ranking item as a "necessity". 


\section{Key points}

- Research on health inequalities among older adults is limited by the lack of a suitable measure of poverty in this age group.

- This study finds that older and younger adults concur on the items needed to avoid poverty.

- Such a measure of "consensual poverty" may be useful for research on health inequalities among adults of all ages

for exploring inequalities in health for all ages, particularly older adults. The time specificity of this measure however must be recognised and it may need to be updated. It is comparatively easy to implement and "incorporates the views of members of the public, rather than judgments by social scientists, about what are the necessities of life in modern Britain". ${ }^{4}$ The PSEOS suggests that identifying people lacking more than one of these "necessities" is a good identifier of those in poverty. It lacks the ability to differentiate between more advantaged groups but could be combined with information on some measurement of income or access to financial resources ${ }^{3}$ to explore gradients in health inequalities.

\section{ACKNOWLEDGEMENTS}

The views expressed in the publication are those of the authors and not those of the Department of Health. We thank Geraldine Barker, Carol Jagger, Graham Martin, Ruth Matthews and Nicky Spiers for their advice as part of the project team and for their comments on the manuscript. The Poverty and Social Exclusion Omnibus Survey data were collected by the Office for National Statistics as part of the ONS Omnibus Survey June 1999 and made available by the UK Data Archive. None of these bears any responsibility for the data, analysis or views expressed in this paper.

The appendix is available on the journal web site (http://www.jech.com/supplemental).

\section{Policy implications}

- This consensual measure of poverty is comparatively easy to implement and "incorporates the views of members of the public, rather than judgments by social scientists, about what are the necessities of life in modern Britain". 4

- It could be used in addition or in place of measures such as social class, to inform and monitor the success of policies aimed at reducing inequalities in health.

\section{Authors' affiliations}

L K Smith, Department of Health Sciences, University of Leicester, Leicester, UK

R M Hancock, Nuffield Community Care Studies Unit, Department of Health Sciences, University of Leicester

Funding: this work was undertaken by Lucy Smith and Ruth Hancock at the University of Leicester who received funding from the Department of Health.

Conflicts of interest: none declared.

Correspondence to: Dr L K Smith, Department of Health Sciences, University of Leicester, 22-28 Princess Road West, Leicester LE1 6TP, UK; lks1@le.ac.uk

Accepted for publication 6 October 2003

\section{REFERENCES}

1 Grundy E, Holt G. The socioeconomic status of older adults: how should we measure it in studies of health inequalities? J Epidemiol Community Health 2001;55:895-904.

2 O'Reilly D. Standard indicators of deprivation: do they disadvantage older people? Age Ageing 2002;31:197-202.

3 Townsend P. Poverty in the United Kingdom. London: Penguin, 1979

4 Gordon D, Adelman L, Ashworth K, et al. Poverty and social exclusion in Britain. York: Joseph Rowntree Foundation, 2000.

5 Gordon D, Pantazis C, Townsend P. Changing necessities of life, 1983-1999, working paper no 3 from the poverty and social exclusion survey of Britain. Bristol: University of Bristol, 2000. 